\title{
Las TIC y su influencia en la calidad del aprendizaje universitario
}

José Rogelio CARRASCo HuamáN ${ }^{1}$

\begin{abstract}
RESUMEN
En este artículo se analizan las tecnologías de la información y comunicación (TIC) y el importante papel que tienen sobre la calidad de la enseñanza universitaria en la Escuela Profesional (EP) de Economía de la Universidad Nacional Mayor de San Marcos (UNMSM). Para ello, se revisará la educación universitaria en países de Latinoamérica y Europa, con el fin de detectar sus fortalezas y usarlas como referencia para la mejora de la calidad de la enseñanza universitaria sanmarquina, sobre todo, de la mencionada EP. Además, se empleará la encuesta (orientada a 120 personas, entre docentes y estudiantes de la Facultad de Ciencias Económicas) como instrumento de recolección de datos, la cual luego será estudiada mediante la escala tipo Likert. Gracias al examen se logrará determinar el grado de influencia de las TIC en el aprendizaje en dicha facultad, así como el beneficio de la aplicación de los factores positivos de la educación universitaria extranjera.
\end{abstract}

Palabras-claves: TIC; tecnologías de la información y comunicación; escala Likert; aprendizaje universitario; calidad educativa.

\section{INTRODUCCIÓN}

Para el ser humano, transferir el conocimiento por medio de la enseñanza y el estudio es un hecho tan antiguo como él mismo. Considerando ese fin, el hombre se ha valido de ejercicios, experiencias, señas, objetos y todo aquello que le ayude a traspasar conocimiento, elemento necesario para que el aprendiz se inicie en algún arte u oficio. Las tecnologías de la información y comunicación (TIC) son una de esas herramientas difusoras de saber, pues permiten dar paso a la educación en forma virtual. De modo que conectan al docente y a los estudiantes, aunque no estén físicamente presentes, permitiéndoles desarrollar actividades autónomas. Actualmente, la computadora y el internet posibilitan crear un aula digital de aprendizaje, a la cual los alumnos pueden acceder de forma remota, economizando energía, tiempo y otros gastos (Cuenca, 2015).

En este artículo se analizará el uso de las TIC en el ámbito de la enseñanza universitaria actual (considerado tanto el contexto mundial, nacional y local), específicamente se examinará su influjo en la Facultad de Ciencias Económicas de la UNMSM y cómo afectan en la calidad del aprendizaje de los estudiantes de pregrado. Además, se plantearán y sugerirán diversas formas de uso e implementación de las TIC en las aulas, laboratorios, auditorios y otros ambientes. Con este estudio se espera sensibilizar a todos aquellos que están inmersos en la enseñanza y la formación del alumnado, así como mejorar la capacitación personal e implementar las nuevas tecnologías (Nakano et al., 2014).

\section{Finlandia}

Este país ocupa el $5 .^{\circ}$ puesto en el mundo en la escala de niveles de aprendizaje, ello según el informe del Programa Internacional para la Evaluación de Estudiantes (Programme for International Student Assessment o PISA). A continuación, a partir del informe PISA y de lo consignado por Álvarez (6 de diciembre de 2016), se mostrarán las características del sistema educativo finlandés:

- La educación es obligatoria y gratuita, por lo tanto, es accesible para todos. Asimismo, debe ser impartida por centros educativos públicos cuando la persona está entre

1 Licenciado en Economía por la Universidad Nacional Mayor de San Marcos. Es docente de la Universidad César Vallejo. Lima, Perú.

E-mail: jcarrascoh@ucv.edu.pe 
los 7 y 16 años. La educación tiene 9 años de duración y cuenta con las TIC actuales (Martí, 6 de junio de 2014).

- Se basa en principios de equidad e igualdad. A su vez, acepta los cambios e innovación en el uso de las TIC (Cabero, 2014).

- Prepara al alumno para poder competir en un mercado internacional y tecnológico (Fuente y Justicia, 2003).

- Un solo profesor se encarga de un grupo desde primer grado (alumnos de 7 años) hasta sexto (estudiantes de 12 años), lo cual ayuda a que el maestro conozca al alumnado y sepa qué técnicas didácticas aplicar para amortiguar alguna debilidad (Bustos y Román, 2011).

- Finlandia cuenta con 14 universidades (de los cuales solo dos son privadas) y 24 escuelas politécnicas. Además, es un país conocido por sus expertos en TIC, biotecnología y diseño, silvicultura, ciencias ambientales, arquitectura y arte (Martí, 6 de junio de 2014).

\section{Alemania}

Esta nación, según Rodrigues (2015), es un ejemplo de uso apropiado de las TIC, pues éstas se han ido incorporando en las empresas. De esa manera, los trabajadores poseen el conocimiento acertado acerca de cómo y con qué fin se incorpora la tecnología en una empresa y en la educación. A su vez, saben de qué manera una organización puede ganar eficiencia, eficacia, competitividad, dinamismo y agilidad. Vale decir que es en las empresas donde las TIC se integran de forma más natural.

\section{Brasil}

De acuerdo con Radio Programas del Perú (30 de agosto de 2012), "Brasil espera convertirse en el tercer mercado de las Tecnologías de la Información y Comunicación”, puesto que "[...] actualmente ocupa el quinto lugar. Brasil espera subir dos peldaños [...] capitalizando su condición de anfitrión del Mundial de Fútbol 2014 y los Juegos Olímpicos 2016" (párrs. 1-2). Este dato refleja que la población brasileña es más receptiva al uso de las nuevas tecnologías.

\section{Perú}

En el Perú, la era de las computadoras personales (PC) inició en 1986, año de ingreso de estos aparatos. A partir de la década de 1990, el costo de las $\mathrm{PC}$ se redujo por la aparición de dispositivos portátiles, como las laptops, notebooks, netbooks, tablets, entre otros (Cuenca, 2015). Debido a lo anterior, el desarrollo tecnológico se extendió a muchas instituciones, sobre todo a las dedicadas a la educación. Así, los beneficios de las nuevas tecnologías y del internet en el campo pedagógico comenzaron a adquirir relevancia. Actualmente, los estudiantes pueden enviar sus trabajos, consultar fuentes y dar respuesta a sus exámenes de manera online; asimismo, tienen disponible abundante información de forma gratuita (Nakano et al, 2014).

Si bien el Perú posee una geografía muy accidentada, los operadores de redes están llegando a lugares remotos, llevando las TIC a espacios donde antes parecía imposible (Barriga, 2009).

\section{Facultad de Ciencias Económicas de la UNMSM}

En 1875 se funda la Facultad de Ciencias Políticas y Administrativas, en una etapa en la que el recurso guanero jugaba un papel importante para la economía nacional. Así, se comenzaron a dictar disciplinas económicas como teoría económica, econometría y matemáticas para economistas; además, se empezaron a utilizar instrumentos como papelógrafos y maquetas. A partir de la década del 2000 esto queda atrás, pues incursionan las TIC en la enseñanza, incorporándose nuevos elementos a las clases (proyectores, software orientado a la economía, econometría, etc.). Estos cambios hicieron posible un notable progreso frente a las modalidades de aprendizaje antiguo (Morales, Trujillo y Raso, 2015).

\section{Metodología}

La presente investigación busca encontrar una relación de correspondencia entre el uso de las TIC y la calidad de aprendizaje de los estudiantes de la Facultad de Ciencias Económicas de la UNMSM. Para ello, se realizó una encuesta, cuyas preguntas y datos obtenidos se muestran en la Tabla 1 (ver anexo).

Cada una de las interrogantes fue elaborada de acuerdo a la necesidad de poder tener cifras que indiquen el grado de empleo actual de las TIC y la manera como estas influyen en la mejora del aprendizaje dentro de la mencionada facultad. Asimismo, estas preguntas permiten ver cómo los docentes y estudiantes van evolucionando y dejando las antiguas herramientas de trabajo académico para pasar a formar parte de la era del conocimiento tecnológico, donde la información es abundante y el tiempo es economizado en relación a los procesos de investigación, aprendizaje y enseñanza.

Este estudio es correlacional, pues permite describir la relación entre las variables, las TIC y su in- 
Tabla 1. Instrumento utilizado y datos obtenidos.

\begin{tabular}{|c|c|c|c|c|c|c|c|c|c|c|c|c|c|c|c|c|c|c|c|c|c|}
\hline Encuesta & P01 & P02 & P03 & P04 & P05 & P06 & P07 & P08 & P09 & P10 & P11 & P12 & P13 & P14 & P15 & P16 & P17 & P18 & P19 & P20 & דٓ \\
\hline $\begin{array}{l}\text { Nunca } \\
=1\end{array}$ & 6 & 1 & 13 & 1 & 1 & 99 & 1 & 1 & 1 & 1 & 1 & 1 & 2 & 11 & 1 & 1 & 1 & 2 & 1 & 1 & \\
\hline $\begin{array}{l}\text { A veces } \\
=2\end{array}$ & 10 & 60 & 17 & 4 & 100 & 18 & 1 & 2 & 3 & 3 & 2 & 2 & 60 & 19 & 2 & 23 & 6 & 0 & 2 & 3 & \\
\hline $\begin{array}{c}\text { Casi } \\
\text { siempre } \\
=3\end{array}$ & 82 & 57 & 86 & 114 & 18 & 1 & 28 & 114 & 98 & 86 & 20 & 2 & 57 & 3 & 101 & 94 & 85 & 110 & 84 & 4 & \\
\hline $\begin{array}{l}\text { Siempre } \\
=4\end{array}$ & 22 & 2 & 4 & 1 & 1 & 2 & 90 & 3 & 18 & 30 & 97 & 115 & 1 & 87 & 16 & 2 & 28 & 8 & 33 & 112 & \\
\hline Varianzas & 0,47 & 0,30 & 0,51 & 0,07 & 0,17 & 0,29 & 0,27 & 0,08 & 0,20 & 0,27 & 0,26 & 0,15 & 0,30 & 1,11 & 0,17 & 0,21 & 0,29 & 0,13 & 0,27 & 0,20 & \\
\hline 1 & 4 & 3 & 3 & 3 & 3 & 2 & 4 & 3 & 3 & 3 & 4 & 4 & 2 & 2 & 3 & 3 & 4 & 3 & 4 & 4 & 64,00 \\
\hline 2 & 1 & 2 & 1 & 3 & 2 & 1 & 3 & 3 & 2 & 2 & 2 & 2 & 2 & 2 & 2 & 2 & 2 & 1 & 2 & 2 & 39,00 \\
\hline 3 & 3 & 2 & 1 & 3 & 2 & 1 & 3 & 2 & 2 & 2 & 1 & 4 & 3 & 2 & 3 & 2 & 3 & 3 & 3 & 1 & 46,00 \\
\hline 4 & 2 & 3 & 1 & 3 & 3 & 1 & 4 & 3 & 4 & 3 & 3 & 4 & 2 & 1 & 3 & 2 & 3 & 4 & 2 & 4 & 55,00 \\
\hline 5 & 3 & 2 & 3 & 3 & 3 & 1 & 4 & 3 & 3 & 3 & 3 & 4 & 2 & 2 & 3 & 2 & 3 & 3 & 3 & 4 & 57,00 \\
\hline 6 & 1 & 2 & 3 & 3 & 3 & 1 & 3 & 3 & 3 & 3 & 3 & 4 & 2 & 2 & 3 & 2 & 3 & 3 & 3 & 4 & 54,00 \\
\hline 7 & 3 & 3 & 2 & 3 & 2 & 1 & 3 & 4 & 3 & 3 & 3 & 4 & 2 & 2 & 3 & 2 & 3 & 4 & 3 & 4 & 57,00 \\
\hline 8 & 3 & 2 & 1 & 3 & 3 & 1 & 3 & 3 & 3 & 3 & 3 & 3 & 2 & 2 & 3 & 2 & 3 & 3 & 1 & 4 & 51,00 \\
\hline 9 & 2 & 2 & 1 & 2 & 2 & 1 & 4 & 3 & 3 & 3 & 3 & 4 & 2 & 2 & 3 & 2 & 3 & 1 & 3 & 4 & 50,00 \\
\hline 10 & 4 & 2 & 3 & 3 & 2 & 4 & 3 & 3 & 4 & 3 & 4 & 4 & 3 & 4 & 3 & 2 & 3 & 3 & 3 & 3 & 63,00 \\
\hline 11 & 3 & 3 & 2 & 3 & 3 & 1 & 3 & 3 & 3 & 3 & 3 & 4 & 2 & 2 & 3 & 2 & 3 & 4 & 3 & 4 & 57,00 \\
\hline 12 & 4 & 2 & 3 & 2 & 2 & 3 & 3 & 4 & 3 & 3 & 3 & 4 & 3 & 4 & 3 & 2 & 3 & 3 & 3 & 4 & 61,00 \\
\hline 13 & 1 & 2 & 3 & 3 & 2 & 1 & 4 & 3 & 4 & 3 & 2 & 4 & 2 & 1 & 3 & 2 & 3 & 3 & 3 & 3 & 52,00 \\
\hline 14 & 2 & 2 & 2 & 2 & 2 & 1 & 4 & 3 & 3 & 4 & 4 & 3 & 3 & 2 & 4 & 2 & 3 & 4 & 3 & 4 & 57,00 \\
\hline 15 & 3 & 3 & 1 & 3 & 2 & 1 & 4 & 4 & 3 & 3 & 4 & 4 & 3 & 2 & 3 & 2 & 3 & 3 & 3 & 4 & 58,00 \\
\hline 16 & 4 & 2 & 4 & 3 & 2 & 1 & 4 & 3 & 3 & 3 & 3 & 4 & 3 & 4 & 3 & 2 & 3 & 3 & 3 & 4 & 61,00 \\
\hline 17 & 3 & 4 & 4 & 3 & 2 & 1 & 4 & 3 & 4 & 3 & 4 & 4 & 3 & 4 & 4 & 4 & 4 & 4 & 4 & 4 & 70,00 \\
\hline 18 & 3 & 3 & 3 & 3 & 3 & 1 & 4 & 3 & 3 & 4 & 3 & 4 & 3 & 4 & 3 & 2 & 3 & 3 & 3 & 3 & 61,00 \\
\hline 19 & 2 & 2 & 3 & 2 & 3 & 1 & 4 & 3 & 3 & 3 & 4 & 4 & 3 & 2 & 3 & 2 & 3 & 3 & 3 & 4 & 57,00 \\
\hline 20 & 4 & 2 & 1 & 3 & 2 & 1 & 4 & 3 & 3 & 3 & 4 & 4 & 2 & 2 & 4 & 2 & 3 & 3 & 3 & 4 & 57,00 \\
\hline 21 & 3 & 2 & 1 & 3 & 3 & 1 & 4 & 3 & 4 & 4 & 4 & 4 & 3 & 3 & 3 & 2 & 3 & 3 & 3 & 4 & 60,00 \\
\hline 22 & 2 & 3 & 1 & 3 & 2 & 1 & 4 & 3 & 3 & 3 & 4 & 4 & 3 & 2 & 3 & 2 & 3 & 3 & 4 & 4 & 57,00 \\
\hline 23 & 2 & 2 & 3 & 3 & 2 & 1 & 4 & 3 & 3 & 3 & 4 & 4 & 3 & 2 & 3 & 2 & 3 & 4 & 3 & 4 & 58,00 \\
\hline 24 & 2 & 2 & 2 & 3 & 2 & 2 & 4 & 3 & 3 & 4 & 4 & 4 & 3 & 2 & 3 & 2 & 3 & 3 & 3 & 4 & 58,00 \\
\hline 25 & 3 & 3 & 3 & 3 & 2 & 2 & 4 & 3 & 3 & 3 & 4 & 4 & 2 & 4 & 3 & 2 & 3 & 3 & 4 & 4 & 62,00 \\
\hline$\ldots$ & 3 & 2 & 2 & 3 & 2 & 1 & 4 & 3 & 3 & 3 & 4 & 4 & 2 & 4 & 3 & 3 & 3 & 3 & 4 & 4 & 60,00 \\
\hline 30 & 3 & 2 & 1 & 3 & 2 & 1 & 4 & 3 & 3 & 4 & 4 & 4 & 3 & 4 & 3 & 3 & 3 & 3 & 4 & 4 & 61,00 \\
\hline 31 & 3 & 2 & 1 & 3 & 2 & 1 & 4 & 3 & 3 & 3 & 4 & 4 & 2 & 2 & 3 & 3 & 3 & 3 & 4 & 4 & 57,00 \\
\hline 32 & 1 & 3 & 3 & 3 & 2 & 1 & 4 & 3 & 3 & 3 & 4 & 4 & 2 & 4 & 3 & 3 & 4 & 3 & 3 & 4 & 60,00 \\
\hline 33 & 3 & 2 & 2 & 3 & 2 & 1 & 4 & 3 & 3 & 4 & 4 & 4 & 2 & 4 & 3 & 3 & 4 & 3 & 4 & 4 & 62,00 \\
\hline$\ldots$ & 3 & 2 & 3 & 3 & 2 & 1 & 4 & 3 & 4 & 3 & 4 & 4 & 3 & 4 & 4 & 3 & 3 & 3 & 3 & 4 & 63,00 \\
\hline 41 & 4 & 3 & 3 & 3 & 2 & 1 & 4 & 3 & 3 & 3 & 4 & 4 & 3 & 4 & 3 & 3 & 3 & 3 & 4 & 4 & 64,00 \\
\hline 42 & 3 & 2 & 2 & 3 & 2 & 1 & 4 & 3 & 3 & 3 & 4 & 4 & 2 & 4 & 4 & 3 & 4 & 3 & 4 & 4 & 62,00 \\
\hline 43 & 3 & 2 & 3 & 3 & 2 & 1 & 4 & 3 & 3 & 3 & 4 & 4 & 3 & 4 & 3 & 3 & 4 & 3 & 3 & 4 & 62,00 \\
\hline 44 & 3 & 2 & 3 & 3 & 2 & 1 & 4 & 3 & 3 & 3 & 4 & 4 & 3 & 4 & 3 & 3 & 4 & 3 & 3 & 4 & 62,00 \\
\hline 45 & 3 & 3 & 3 & 3 & 2 & 1 & 4 & 3 & 3 & 3 & 4 & 4 & 3 & 4 & 4 & 3 & 4 & 3 & 4 & 4 & 65,00 \\
\hline 46 & 3 & 2 & 2 & 3 & 2 & 1 & 4 & 3 & 3 & 3 & 4 & 4 & 2 & 4 & 3 & 3 & 4 & 3 & 3 & 4 & 60,00 \\
\hline 47 & 3 & 2 & 3 & 3 & 2 & 1 & 4 & 3 & 3 & 3 & 4 & 4 & 3 & 4 & 3 & 3 & 3 & 3 & 3 & 4 & 61,00 \\
\hline 48 & 3 & 2 & 3 & 3 & 2 & 1 & 4 & 3 & 3 & 4 & 4 & 4 & 2 & 4 & 3 & 3 & 4 & 3 & 3 & 4 & 62,00 \\
\hline$\ldots$ & 4 & 3 & 2 & 3 & 2 & 1 & 4 & 3 & 3 & 3 & 4 & 4 & 2 & 4 & 3 & 3 & 3 & 3 & 3 & 4 & 61,00 \\
\hline 59 & 3 & 2 & 3 & 3 & 2 & 1 & 4 & 3 & 3 & 3 & 4 & 4 & 3 & 4 & 4 & 3 & 4 & 3 & 3 & 4 & 63,00 \\
\hline 60 & 4 & 3 & 3 & 3 & 2 & 1 & 4 & 3 & 3 & 4 & 4 & 4 & 3 & 4 & 3 & 3 & 4 & 3 & 4 & 4 & 66,00 \\
\hline 61 & 3 & 3 & 3 & 3 & 2 & 1 & 4 & 3 & 3 & 3 & 4 & 4 & 2 & 4 & 3 & 3 & 3 & 3 & 3 & 4 & 61,00 \\
\hline 62 & 4 & 2 & 3 & 3 & 2 & 1 & 4 & 3 & 3 & 3 & 4 & 4 & 2 & 4 & 3 & 3 & 3 & 3 & 3 & 4 & 61,00 \\
\hline 63 & 3 & 3 & 3 & 3 & 2 & 1 & 4 & 3 & 3 & 3 & 4 & 4 & 3 & 4 & 4 & 3 & 3 & 3 & 3 & 4 & 63,00 \\
\hline 64 & 3 & 3 & 3 & 3 & 2 & 1 & 4 & 3 & 3 & 3 & 4 & 4 & 2 & 4 & 3 & 3 & 3 & 3 & 4 & 4 & 62,00 \\
\hline
\end{tabular}




\begin{tabular}{|c|c|c|c|c|c|c|c|c|c|c|c|c|c|c|c|c|c|c|c|c|c|}
\hline Encuesta & P01 & P02 & P03 & P04 & P05 & P06 & P07 & P08 & P09 & P10 & P11 & P12 & P13 & P14 & P15 & P16 & P17 & P18 & P19 & P20 & 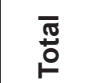 \\
\hline 65 & 3 & 3 & 3 & 3 & 2 & 1 & 4 & 3 & 3 & 3 & 4 & 4 & 3 & 4 & 3 & 3 & 3 & 3 & 4 & 4 & 63,00 \\
\hline 66 & 3 & 3 & 3 & 3 & 2 & 1 & 4 & 3 & 3 & 3 & 4 & 4 & 2 & 4 & 3 & 3 & 3 & 3 & 4 & 4 & 62,00 \\
\hline 67 & 3 & 3 & 3 & 3 & 3 & 1 & 4 & 3 & 3 & 3 & 4 & 4 & 2 & 4 & 3 & 3 & 4 & 3 & 3 & 4 & 63,00 \\
\hline 68 & 4 & 3 & 3 & 3 & 2 & 1 & 4 & 3 & 3 & 3 & 4 & 4 & 3 & 4 & 3 & 3 & 4 & 3 & 4 & 4 & 65,00 \\
\hline 69 & 3 & 3 & 3 & 3 & 2 & 1 & 4 & 3 & 3 & 3 & 4 & 4 & 2 & 4 & 3 & 3 & 4 & 3 & 4 & 4 & 63,00 \\
\hline 70 & 3 & 3 & 3 & 3 & 2 & 1 & 4 & 3 & 3 & 3 & 4 & 4 & 3 & 4 & 3 & 3 & 4 & 3 & 3 & 4 & 63,00 \\
\hline 71 & 3 & 3 & 3 & 3 & 2 & 4 & 4 & 3 & 3 & 4 & 4 & 4 & 2 & 4 & 3 & 3 & 4 & 3 & 3 & 4 & 66,00 \\
\hline 72 & 4 & 3 & 3 & 3 & 2 & 1 & 4 & 3 & 3 & 4 & 4 & 4 & 3 & 4 & 3 & 3 & 2 & 3 & 3 & 4 & 63,00 \\
\hline 73 & 3 & 2 & 3 & 3 & 2 & 1 & 4 & 3 & 4 & 3 & 4 & 4 & 3 & 4 & 3 & 3 & 2 & 3 & 3 & 4 & 61,00 \\
\hline 74 & 3 & 3 & 3 & 3 & 2 & 1 & 4 & 3 & 4 & 4 & 4 & 4 & 2 & 4 & 3 & 3 & 4 & 3 & 3 & 4 & 64,00 \\
\hline 75 & 4 & 3 & 3 & 3 & 2 & 1 & 4 & 3 & 3 & 4 & 4 & 4 & 2 & 4 & 3 & 3 & 3 & 3 & 3 & 4 & 63,00 \\
\hline 76 & 4 & 3 & 3 & 3 & 2 & 1 & 4 & 3 & 3 & 4 & 4 & 4 & 3 & 4 & 4 & 3 & 3 & 3 & 3 & 4 & 65,00 \\
\hline 77 & 3 & 2 & 3 & 3 & 2 & 2 & 4 & 3 & 3 & 4 & 4 & 4 & 3 & 4 & 4 & 3 & 3 & 3 & 3 & 4 & 64,00 \\
\hline 78 & 3 & 2 & 2 & 3 & 2 & 2 & 4 & 3 & 4 & 4 & 4 & 4 & 2 & 4 & 3 & 3 & 3 & 3 & 3 & 4 & 62,00 \\
\hline 79 & 3 & 2 & 3 & 3 & 2 & 2 & 4 & 3 & 3 & 3 & 4 & 4 & 3 & 4 & 3 & 3 & 3 & 3 & 3 & 4 & \begin{tabular}{|l|}
62,00 \\
\end{tabular} \\
\hline 80 & 3 & 2 & 3 & 3 & 2 & 1 & 4 & 3 & 3 & 4 & 4 & 4 & 3 & 4 & 3 & 3 & 3 & 3 & 3 & 4 & 62,00 \\
\hline 81 & 3 & 3 & 3 & 3 & 2 & 2 & 4 & 3 & 3 & 4 & 4 & 4 & 2 & 4 & 3 & 3 & 3 & 3 & 3 & 4 & 63,00 \\
\hline 82 & 3 & 3 & 3 & 3 & 2 & 1 & 4 & 3 & 3 & 4 & 4 & 4 & 3 & 3 & 3 & 3 & 3 & 3 & 3 & 4 & \begin{tabular}{|l|l|}
62,00 \\
\end{tabular} \\
\hline 83 & 3 & 2 & 3 & 3 & 2 & 1 & 4 & 3 & 3 & 4 & 4 & 4 & 2 & 3 & 4 & 3 & 3 & 3 & 3 & 4 & 61,00 \\
\hline 84 & 3 & 3 & 3 & 3 & 2 & 1 & 4 & 3 & 4 & 3 & 4 & 4 & 3 & 4 & 3 & 3 & 4 & 3 & 4 & 4 & 65,00 \\
\hline 85 & 3 & 2 & 2 & 3 & 2 & 1 & 4 & 3 & 4 & 4 & 4 & 4 & 3 & 4 & 3 & 3 & 3 & 3 & 4 & 4 & \begin{tabular}{|l|}
63,00 \\
\end{tabular} \\
\hline 86 & 4 & 3 & 3 & 3 & 2 & 2 & 4 & 3 & 3 & 4 & 4 & 4 & 3 & 4 & 3 & 3 & 3 & 3 & 4 & 4 & 66,00 \\
\hline 87 & 3 & 2 & 3 & 3 & 2 & 1 & 4 & 3 & 3 & 3 & 4 & 4 & 3 & 4 & 3 & 3 & 3 & 3 & 4 & 4 & 62,00 \\
\hline 88 & 3 & 3 & 3 & 3 & 2 & 2 & 4 & 3 & 3 & 4 & 4 & 4 & 3 & 4 & 3 & 3 & 3 & 3 & 3 & 4 & 64,00 \\
\hline 89 & 4 & 2 & 3 & 3 & 2 & 2 & 4 & 3 & 3 & 3 & 4 & 4 & 3 & 4 & 3 & 3 & 3 & 3 & 4 & 4 & 64,00 \\
\hline 90 & 3 & 2 & 3 & 3 & 2 & 1 & 4 & 3 & 3 & 3 & 4 & 4 & 2 & 4 & 3 & 3 & 3 & 3 & 4 & 4 & 61,00 \\
\hline 91 & 3 & 2 & 3 & 3 & 2 & 2 & 4 & 3 & 3 & 3 & 4 & 4 & 2 & 4 & 3 & 3 & 3 & 3 & 3 & 4 & 61,00 \\
\hline 92 & 4 & 3 & 3 & 3 & 2 & 2 & 4 & 3 & 4 & 3 & 4 & 4 & 2 & 4 & 3 & 3 & 2 & 3 & 3 & 4 & 63,00 \\
\hline 93 & 3 & 3 & 3 & 3 & 2 & 2 & 4 & 3 & 3 & 3 & 4 & 4 & 2 & 4 & 3 & 3 & 3 & 3 & 3 & 4 & 62,00 \\
\hline 94 & 3 & 2 & 3 & 3 & 2 & 2 & 4 & 3 & 3 & 4 & 4 & 4 & 2 & 4 & 3 & 3 & 3 & 3 & 3 & 4 & 62,00 \\
\hline$\ldots$ & 3 & 3 & 3 & 3 & 2 & 1 & 3 & 3 & 3 & 3 & 3 & 4 & 2 & 4 & 3 & 3 & 3 & 3 & 4 & 4 & 60,00 \\
\hline 106 & 3 & 2 & 3 & 3 & 2 & 2 & 3 & 3 & 3 & 3 & 4 & 4 & 2 & 4 & 4 & 3 & 3 & 3 & 3 & 4 & 61,00 \\
\hline 107 & 3 & 3 & 3 & 3 & 2 & 1 & 3 & 3 & 3 & 3 & 4 & 4 & 2 & 4 & 3 & 3 & 3 & 3 & 3 & 4 & 60,00 \\
\hline 108 & 3 & 3 & 3 & 3 & 2 & 1 & 3 & 3 & 3 & 3 & 4 & 4 & 2 & 1 & 3 & 1 & 3 & 3 & 3 & 4 & 55,00 \\
\hline 109 & 4 & 3 & 3 & 3 & 2 & 1 & 3 & 3 & 4 & 3 & 4 & 4 & 2 & 4 & 3 & 3 & 3 & 3 & 3 & 4 & 62,00 \\
\hline 110 & 3 & 3 & 3 & 3 & 2 & 1 & 3 & 3 & 3 & 3 & 4 & 4 & 3 & 4 & 3 & 3 & 3 & 3 & 3 & 4 & 61,00 \\
\hline 111 & 3 & 2 & 3 & 3 & 2 & 1 & 3 & 3 & 4 & 3 & 3 & 4 & 2 & 2 & 3 & 3 & 3 & 3 & 3 & 4 & 57,00 \\
\hline 112 & 3 & 2 & 3 & 3 & 3 & 1 & 3 & 1 & 3 & 3 & 3 & 4 & 1 & 1 & 3 & 3 & 2 & 3 & 3 & 4 & 52,00 \\
\hline 113 & 2 & 4 & 3 & 1 & 2 & 1 & 3 & 3 & 3 & 3 & 3 & 4 & 2 & 1 & 3 & 3 & 3 & 3 & 3 & 4 & \begin{tabular}{|l|}
54,00 \\
\end{tabular} \\
\hline 114 & 3 & 2 & 2 & 3 & 2 & 1 & 3 & 3 & 3 & 3 & 4 & 4 & 2 & 1 & 3 & 3 & 3 & 3 & 3 & 4 & 55,00 \\
\hline 115 & 3 & 3 & 3 & 3 & 2 & 1 & 3 & 3 & 3 & 3 & 3 & 4 & 2 & 1 & 3 & 3 & 3 & 3 & 3 & 4 & 56,00 \\
\hline 116 & 3 & 3 & 2 & 3 & 4 & 2 & 3 & 3 & 3 & 2 & 3 & 4 & 2 & 1 & 2 & 3 & 1 & 3 & 3 & 2 & \begin{tabular}{|l|}
52,00 \\
\end{tabular} \\
\hline 117 & 3 & 3 & 4 & 3 & 3 & 1 & 1 & 3 & 3 & 3 & 3 & 4 & 2 & 2 & 3 & 3 & 3 & 3 & 3 & 4 & 57,00 \\
\hline 118 & 3 & 3 & 3 & 3 & 1 & 1 & 3 & 3 & 3 & 3 & 3 & 1 & 4 & 1 & 1 & 3 & 3 & 3 & 3 & 2 & 50,00 \\
\hline 119 & 1 & 3 & 1 & 3 & 3 & 1 & 4 & 2 & 1 & 1 & 3 & 4 & 3 & 1 & 3 & 3 & 3 & 3 & 3 & 4 & 50,00 \\
\hline 120 & 3 & 1 & 2 & 4 & 2 & 1 & 2 & 3 & 2 & 3 & 4 & 2 & 2 & 1 & 3 & 3 & 3 & 3 & 3 & 4 & 51,00 \\
\hline Moda & 3,0 & 2,0 & 3,0 & 3,0 & 2,0 & 1,0 & 4,0 & 3,0 & 3,0 & 3,0 & 4,0 & 4,0 & 2,0 & 4,0 & 3,0 & 3,0 & 3,0 & 3,0 & 3,0 & 4,0 & \\
\hline Mediana & 3,0 & 2,0 & 3,0 & 3,0 & 2,0 & 1,0 & 4,0 & 3,0 & 3,0 & 3,0 & 4,0 & 4,0 & 2,0 & 4,0 & 3,0 & 3,0 & 3,0 & 3,0 & 3,0 & 4,0 & \\
\hline 1. ${ }^{\circ}$ cuartil & 1,00 & & & & & & & & & & & & & & & & & & & & \\
\hline $3 .^{\circ}$ cuartil & 4,00 & & & & & & & & & & & & & & & & & & & & \\
\hline Covarianza & 0,13 & & & & & & & & & & & & & & & & & & & & \\
\hline
\end{tabular}

Fuente: Elaboración propia. 
fluencia; además, es transversal, porque hace una recolección de datos. La muestra de la investigación tomó como universo a los estudiantes y docentes de la Facultad de Ciencias Económicas de la UNMSM, considerando un total de 60 docentes y 1800 estudiantes. Nuestro prototipo equivale a un $17,61 \%$ del universo, con un margen de error del $5 \%$ y un nivel de confianza del $95 \%$; en tal sentido, se consideró para la encuesta a 120 individuos, entre profesores y alumnos.

\section{Fundamentación y formulación de la hipótesis}

\section{Hipótesis general}

La aplicación de las TIC adecuadas influye en la calidad del aprendizaje universitario.

\section{Hipótesis específicas}

- Las TIC adecuadas mejoran la calidad del aprendizaje universitario.

- El nivel de la calidad del aprendizaje influye en la formación de los alumnos universitarios.

\section{RESULTADOS}

La Tabla 1, que figura en el anexo, muestra los resultados de las 120 encuestas realizadas.

\section{Análisis de fiabilidad}

Para el presente estudio se empleará el alfa de Cronbach, pues — según Ledesma (2004)— este permite determinar la consistencia de una encuesta y su confiabilidad. Así, se encontró el valor 0,744, el cual está dentro del rango 0,7-0,8, y cuya interpretación define al cuestionario empleado como un instrumento confiable. El resultado se obtuvo mediante el sistema informático IBM SPSS Statistics 24.0. En la Tabla 2, se aprecian los resultados de las estadísticas de fiabilidad por medio del análisis de Cronbach.

Tabla 2. Análisis de Cronbach.

\begin{tabular}{|l|l|}
\hline \multicolumn{2}{|c|}{ Estadísticas de fiabilidad } \\
\hline Alfa de Cronbach & N. ${ }^{\circ}$ de elementos \\
\hline 0,744 & 20 \\
\hline
\end{tabular}

Fuente: Elaboración propia.

\section{Análisis factorial exploratorio}

Se realizó un análisis factorial exploratorio de componentes principales con rotación Varimax. Además, la prueba de esfericidad de Barlett permitió determinar si la matriz era factorizable. Los resultados se muestran en la Tabla 3.
Tabla 3. Prueba Kaiser-Meyer-Olkin y Barlett.

\begin{tabular}{|l|l|c|}
\hline \multicolumn{2}{|l|}{$\begin{array}{l}\text { Medida Kaiser-Meyer-Olkin de adecuación de } \\
\text { muestreo }\end{array}$} & 0,679 \\
\hline $\begin{array}{l}\text { Prueba de } \\
\text { esfericidad de } \\
\text { Bartlett }\end{array}$ & Aproximación chi-cuadrado & 616,242 \\
\cline { 2 - 3 } & Grados de libertad & 190 \\
\cline { 2 - 3 } & Significancia & 0,000 \\
\hline
\end{tabular}

Fuente: SPSS.

Para la muestra de esta investigación, el índice de Kaiser-Meyer-Olkin (KMO) es de 0,679. Asimismo, fue recomendable hacer el análisis factorial, pues el resultado es mayor a 0,500. La prueba de elasticidad de Barlett resulta altamente significativa $(p=0,000)$, lo que significa que existen relaciones estadísticas relevantes entre algunas variables.

\section{Análisis de hipótesis}

Siguiendo a Cánovas, Martínez, Pérez y Tuya (2009), se utilizó el programa Excel para calcular el coeficiente de correlación para la hipótesis general, obteniéndose un valor de 0,543. Esto indica que la hipótesis es válida. Por lo tanto, se tiene lo siguiente:

- El cálculo del coeficiente de correlación para la hipótesis general da como resultado: $D=0,543$.

- El cálculo del coeficiente de correlación para la hipótesis específica (vinculada a las TIC) da como resultado: $D=0,702$. Esto señala que la hipótesis específica es válida.

- El cálculo del coeficiente de correlación para la hipótesis específica (nivel de aprendizaje) da como resultado: $D=0,612$. Esto indica que la hipótesis específica es válida.

- En todos los casos se obtuvo un resultados $>0,5$. Esto indica que hay correlación entre las variables, confirmando las hipótesis planteadas.

A continuación, se registran las etapas que se siguieron durante el proceso: la selección de la muestra, la elaboración del instrumento, la fabricación de la encuesta para docentes y estudiantes, la impresión y revisión del instrumento, la gestión de los permisos respectivos ante el decano y director de la Escuela Profesional de Economía de la UNMSM, la toma de la muestra con el apoyo de cuatro estudiantes de la Universidad César Vallejo (UCV), la digitación de datos al programa Excel, el análisis estadístico descriptivo mediante la escala Likert, la elaboración de los resultados y la redacción del informe. 


\section{CONCLUSIONES}

- Se contrastaron las hipótesis planteadas con los resultados obtenidos en la encuesta tomada a estudiantes y docentes de la Facultad de Ciencias Económicas de la UNMSM, donde se emplearon las estadísticas de consistencia (alfa de Cronbach) y el coeficiente correlación de Spearman para intervalos de clase. Asimismo, se usaron las estadísticas descriptivas a través de la moda, la mediana y los cuartiles.

- Como se aprecia en la Tabla 1 (ver anexo), se consideraron las variables TIC y la calidad de aprendizaje. A partir de ello, se concluye que el uso del correo electrónico por parte de docentes y estudiantes se ha vuelto una necesidad. Además, la enseñanza presencial sigue siendo más aprovechada y brinda mejores resultados. Por otro lado, el proyector de video resulta indispensable dentro de la actividad didáctica y formativa.

\section{Recomendaciones}

- Solicitar que los docentes estén actualizados en el uso de los software educativos.

- Incrementar el uso de las videoconferencias.

- Utilizar con más frecuencia los exámenes online.

- Mejorar el filtro de la información que se proporciona a los estudiantes.

- Incrementar la difusión de las nuevas tecnologías entre los alumnos.

- Aumentar la atención en el presupuesto destinado al sector educación por parte del Estado peruano.

\section{REFERENCIAS}

[1] Barriga, C. (2009). Investigación Educacional A. Texto autoinstructivo. Lima, Perú: Universidad Nacional Mayor de San Marcos.

[2] Bustos, A. y Román M. (2011). La importancia de evaluar la incorporación y el uso de las TIC en educación. Revista Iberoamericana de Evaluación Educativa, 4(2), 3-7. Recuperado de https://revistas.uam.es/index.php/riee/ article/view/4452/4879

[3] Cabero, J. (2014). Formación del profesorado universitario en TIC. Aplicación del método Delphi para la selección de los contenidos formativos. Educación XX1, 17(1), 111-132.
Recuperado de http://revistas.uned.es/index. php/educacionXX1/article/view/10707/11301

[4] Cánovas, A., Martínez, R., Pérez, A. y Tuya, L. (2009). El coeficiente de correlación de los rangos de Spearman. Caracterización. Revista Habanera de Ciencias Médicas, 8(2). Recuperado de http://scielo.sld.cu/ scielo.php?script=sci_arttext\&pid=S1729519X2009000200017

[5] Cuenca, R. (ed.) (2015). La educación universitaria en el Perú. Democracia, expansión y desigualdades. Lima, Perú: Instituto de Estudios Peruanos.

[6] Fuente, J. de la y Justicia, F. (2003). Escala de estrategias de aprendizaje ACRAAbreviada para alumnos universitarios. Revista Electrónica de Investigación Psicoeducativa y Psicopedagógica, 1(2), 139-158. Recuperado de http://www.investigacion-psicopedagogica. org/revista/articulos/2/espannol/Art_2_16.pdf

[7] Álvarez, P. (6 de diciembre de 2016). Informe PISA. La educación española se estanca en ciencias y matemáticas y mejora levemente en lectura. El País. Recuperado de https://elpais.com/politica/2016/12/05/ actualidad/1480950645_168779.html.

[8] Ledesma, R. (2004). AlphaCl: un programa de cálculo de intervalos de confianza para el coeficiente alfa de Cronbach. Psico-USF, 9(1), 31-37. Recuperado de http://pepsic.bvsalud. org/pdf/psicousf/v9n1/v9n1a05.pdf

[9] Martí, J. (6 de junio de 2014). Las TIC en Finlandia. Xarxatix. Recuperado de http://www. xarxatic.com/las-tic-en-finlandia/

[10] Morales, M., Trujillo, J. y Raso, F. (2015). Percepciones acerca de la integración de las TIC en el proceso de enseñanza-aprendizaje de la universidad. Pixel-Bit. Revista de Medios y Educación, (46), 103-117. Recuperado de https://idus.us.es/xmlui/bitstream/ handle/11441/45403/PERCEPCIONES $\% 20$ A C E R C A \% 20 D E \% 20 L A \% 20 INTEGRACI\%C3\%93N\%20DE\%20LAS\%20 TIC\%20EN.pdf?sequence=1\&isAllowed $=y$

[11] Nakano T., Garret, P., Vásquez, A. y Mija, Á. (2014). La integración de las TIC en la educación superior: reflexiones y aprendizajes a partir de la experiencia PUCP. Blanco \& Negro. Revista sobre Docencia Universitaria, 4(2), 65-76. Recuperado de http://revistas. pucp.edu.pe/index.php/enblancoynegro/ article/view/8936/9344 
[12] Rodrigues, L. M. A. R. (2015). Os jogos como método de aprendizagem de vocabulário nas aulas de espanhol como língua estrangeira (ELE). (Tesis doctoral). Recuperado de https:// repositorio.ucp.pt/handle/10400.14/18809.
[13] Radio Programas del Perú (30 de agosto de 2012). Brasil quiere convertirse en el tercer mercado mundial de las TIC. Recuperado de https://rpp.pe/lima/actualidad/brasil-quiereconvertirse-en-el-tercer-mercado-mundial-delas-tic-noticia-517195. 


\section{The influence of Information and Communication Technologies (ICTs) on the quality of university learning}

José Rogelio CARrasco Huamán ${ }^{1}$

\begin{abstract}
This paper analyzes information and communication technologies (ICTs) and their important role on the quality of university education at the Department of Economics of the Universidad Nacional Mayor de San Marcos (UNMSM). To this end, university education in Latin American and European countries is studied in order to identify strengths and use them as reference points to improve the quality of education at UNMSM, especially at said department. A questionnaire, directed at 120 respondents, including professors and students of the School of Economic Sciences, is used as a data collection instrument, which is then analyzed using the Likert scale. This analysis makes it possible to determine the degree of influence ICTs have on the quality of learning at said school, as well as the benefits of implementing the identified strengths of foreign university education.
\end{abstract}

Keywords: ICT; communication and information technologies; Likert scale; university learning; quality of education.

\section{INTRODUCTION}

The transfer of knowledge by means of teaching and study is a fact as old as humankind itself. To this end, human beings have used exercises, experiences, signs, objects and all other means to enable them to transfer knowledge, the necessary feature for an apprentice to begin his training in a particular art or trade. Information and communication technologies (ICTs) are one of those knowledge dissemination tools, as they enable education in a virtual form. Thus, they connect teachers and students, even if not physically present, allowing them to perform independent activities. Nowadays, computers and the Internet make it possible to create digital classrooms, which students can access remotely, saving energy, time and other costs (Cuenca, 2015).

This paper analyzes the usage of ICTs within the framework of current university education (considering global, national and local contexts), specifically their influence on the School of Economic Sciences of the UNMSM and how they affect the quality of undergraduate student learning. In addition, various forms of use and implementation of ICT tools in classrooms, laboratories, auditoriums and other settings are suggested. This paper attempts to educate all those involved in the education and training of students, as well as improve individual training and implement new technologies (Nakano et al., 2014).

\section{Finland}

According to the Program for International Student Assessment (PISA) report, this country ranks fifth worldwide in learning levels. Based on the PISA report and information provided by Álvarez (December 6, 2016), the characteristics of the Finnish education system are presented:

- Education is compulsory and free, therefore, it is accessible to all. Likewise, children aged seven to 16 must be educated at public educational establishments. Basic education lasts nine years and includes current ICTs (Martí, June 6, 2014).

1 Licenciado* in Economics from the Universidad Nacional Mayor de San Marcos. Professor at the Universidad César Vallejo. Lima, Peru.

E-mail: jcarrascoh@ucv.edu.pe

*In Peru, Licenciatura or Título is a professional title granted after having defended a thesis or passing a course in accordance with the university's regulations once the undergraduate program has been completed. 
- It is based on principles of equity and equality. At the same time, it accepts changes and innovation in the use of ICTs (Cabero, 2014).

- It prepares the pupil to compete in an international and technological market (Fuente \& Justicia, 2003).

- The same teacher teaches a group from grade 1 (pupils aged 7 ) to 6 (pupils aged 12), which helps the teacher to know the students and what teaching techniques to apply in order to lessen any weakness (Bustos \& Román, 2011).

- There are 14 universities (of which only two are private) and 24 polytechnic schools in Finland. In addition, this country is known for its experts in ICTs, biotechnology and design, silviculture, environmental sciences, architecture and art (Martí, June 6, 2014).

\section{Germany}

As per Rodrigues (2015), this nation is an example of the proper use of ICT tools, as these have been incorporated into companies. Accordingly, workers are aware of how and for what purpose technology is integrated into a company and into education. At the same time, they know how technology in organizations can result in efficiency, efficacy, competitiveness, dynamism and agility improvements. It is worth noting that ICTs integrate more naturally into companies.

\section{Brazil}

According to Radio Programas del Perú (August 30, 2012), "Brasil espera convertirse en el tercer mercado de las Tecnologías de la Información y Comunicación" [Brazil hopes to become the third Information and Communication Technology market], since "[...] actualmente ocupa el quinto lugar. Brasil espera subir dos peldaños [...] capitalizando su condición de anfitrión del Mundial de Fútbol 2014 y los Juegos Olímpicos 2016" [it currently ranks fifth. Brazil hopes to climb two places ... capitalizing on its position as host of the 2014 World Cup and 2016 Olympic Games] (para. 1-2). This data reflects the fact that Brazilian population is more receptive to the use of new technologies.

\section{Peru}

In Peru, the era of personal computers (PC) began in 1986, the year in which these devices entered the market. Since the beginning of the 1990s, the cost of PCs has been dropping due to the advent of portable devices such as laptops, notebooks, netbooks, tablets, among others (Cuenca, 2015). As a result, technological development spread to several establishments, especially those devoted to education. Thus, the benefits obtained from the implementation of new technologies and the Internet in the educational field began to gain relevance. Nowadays, students can send their assignments, consult sources and take exams online. Pupils also have ample information available for free (Nakano et al., 2014).

Although Peru has a difficult topography, network service providers are reaching remote areas, taking ICTs to places that once seemed impossible to reach (Barriga, 2009).

\section{School of Economic Sciences of UNMSM}

The Facultad de Ciencias Políticas y Administrativas [School of Political and Administrative Sciences] was founded in 1875, at a time during which the guano resource played a major role in the country's economy. Accordingly, economic disciplines as economic theory, econometrics, and mathematics for economists began to be taught, and instruments such as flip charts and scale models began to be used. At the beginning of the 2000s this was left behind as the ICTs moved into education, incorporating new elements to classes (projectors, economy-oriented software, etc.). These changes made possible significant progress in education compared with more traditional types of learning (Morales, Trujillo \& Raso).

\section{Methodology}

This paper aims at finding a correspondence relationship between the use of ICTs and the quality of learning for School of Economic Sciences students at UNMSM. For this purpose, a survey by questionnaire was conducted. Said questionnaire and the data obtained are exhibited in Table 1 (see Annex).

Each question was elaborated according to the need of obtaining figures that would indicate the extent of the current use of ICTs and the manner in which these influence the improvement of learning within the school. Additionally, these questions make it possible to observe how professors and pupils are evolving and leaving behind the traditional tools associated with academic work to become part of the technology age, where information is abundant and time is economized in relation to research, learning and teaching processes.

This is a correlational study, as it allows the description of the relationship between variables, the ICTs and their influence; furthermore, it is cross-sectional, because data is collected. The research sample 
Table 1. Instrument used and obtained data.

\begin{tabular}{|c|c|c|c|c|c|c|c|c|c|c|c|c|c|c|c|c|c|c|c|c|c|}
\hline $\begin{array}{l}\text { Question- } \\
\text { naire }\end{array}$ & Q01 & Q02 & Q03 & Q04 & Q05 & Q06 & Q07 & Q08 & Q09 & Q10 & Q11 & Q12 & Q13 & Q14 & Q15 & Q16 & Q17 & Q18 & Q19 & Q20 & సٓํํ \\
\hline Never $=1$ & 6 & 1 & 13 & 1 & 1 & 99 & 1 & 1 & 1 & 1 & 1 & 1 & 2 & 11 & 1 & 1 & 1 & 2 & 1 & 1 & \\
\hline $\begin{array}{l}\text { Someti- } \\
\text { mes }=2\end{array}$ & 10 & 60 & 17 & 4 & 100 & 18 & 1 & 2 & 3 & 3 & 2 & 2 & 60 & 19 & 2 & 23 & 6 & 0 & 2 & 3 & \\
\hline $\begin{array}{l}\text { Usually } \\
\quad=3\end{array}$ & 82 & 57 & 86 & 114 & 18 & 1 & 28 & 114 & 98 & 86 & 20 & 2 & 57 & 3 & 101 & 94 & 85 & 110 & 84 & 4 & \\
\hline $\begin{array}{c}\text { Always } \\
=4\end{array}$ & 22 & 2 & 4 & 1 & 1 & 2 & 90 & 3 & 18 & 30 & 97 & 115 & 1 & 87 & 16 & 2 & 28 & 8 & 33 & 112 & \\
\hline Variances & 0.47 & 0.30 & 0.51 & 0.07 & 0.17 & 0.29 & 0.27 & 0.08 & 0.20 & 0.27 & 0.26 & 0.15 & \begin{tabular}{|l|}
0.30 \\
\end{tabular} & 1.11 & \begin{tabular}{|l|}
0.17 \\
\end{tabular} & 0.21 & 0.29 & 0.13 & 0.27 & 0.20 & \\
\hline 1 & 4 & 3 & 3 & 3 & 3 & 2 & 4 & 3 & 3 & 3 & 4 & 4 & 2 & 2 & 3 & 3 & 4 & 3 & 4 & 4 & 64.00 \\
\hline 2 & 1 & 2 & 1 & 3 & 2 & 1 & 3 & 3 & 2 & 2 & 2 & 2 & 2 & 2 & 2 & 2 & 2 & 1 & 2 & 2 & 39.00 \\
\hline 3 & 3 & 2 & 1 & 3 & 2 & 1 & 3 & 2 & 2 & 2 & 1 & 4 & 3 & 2 & 3 & 2 & 3 & 3 & 3 & 1 & 46.00 \\
\hline 4 & 2 & 3 & 1 & 3 & 3 & 1 & 4 & 3 & 4 & 3 & 3 & 4 & 2 & 1 & 3 & 2 & 3 & 4 & 2 & 4 & 55.00 \\
\hline 5 & 3 & 2 & 3 & 3 & 3 & 1 & 4 & 3 & 3 & 3 & 3 & 4 & 2 & 2 & 3 & 2 & 3 & 3 & 3 & 4 & 57.00 \\
\hline 6 & 1 & 2 & 3 & 3 & 3 & 1 & 3 & 3 & 3 & 3 & 3 & 4 & 2 & 2 & 3 & 2 & 3 & 3 & 3 & 4 & 54.00 \\
\hline 7 & 3 & 3 & 2 & 3 & 2 & 1 & 3 & 4 & 3 & 3 & 3 & 4 & 2 & 2 & 3 & 2 & 3 & 4 & 3 & 4 & 57.00 \\
\hline 8 & 3 & 2 & 1 & 3 & 3 & 1 & 3 & 3 & 3 & 3 & 3 & 3 & 2 & 2 & 3 & 2 & 3 & 3 & 1 & 4 & 51.00 \\
\hline 9 & 2 & 2 & 1 & 2 & 2 & 1 & 4 & 3 & 3 & 3 & 3 & 4 & 2 & 2 & 3 & 2 & 3 & 1 & 3 & 4 & 50.00 \\
\hline 10 & 4 & 2 & 3 & 3 & 2 & 4 & 3 & 3 & 4 & 3 & 4 & 4 & 3 & 4 & 3 & 2 & 3 & 3 & 3 & 3 & 63.00 \\
\hline 11 & 3 & 3 & 2 & 3 & 3 & 1 & 3 & 3 & 3 & 3 & 3 & 4 & 2 & 2 & 3 & 2 & 3 & 4 & 3 & 4 & 57.00 \\
\hline 12 & 4 & 2 & 3 & 2 & 2 & 3 & 3 & 4 & 3 & 3 & 3 & 4 & 3 & 4 & 3 & 2 & 3 & 3 & 3 & 4 & 61.00 \\
\hline 13 & 1 & 2 & 3 & 3 & 2 & 1 & 4 & 3 & 4 & 3 & 2 & 4 & 2 & 1 & 3 & 2 & 3 & 3 & 3 & 3 & 52.00 \\
\hline 14 & 2 & 2 & 2 & 2 & 2 & 1 & 4 & 3 & 3 & 4 & 4 & 3 & 3 & 2 & 4 & 2 & 3 & 4 & 3 & 4 & 57.00 \\
\hline 15 & 3 & 3 & 1 & 3 & 2 & 1 & 4 & 4 & 3 & 3 & 4 & 4 & 3 & 2 & 3 & 2 & 3 & 3 & 3 & 4 & 58.00 \\
\hline 16 & 4 & 2 & 4 & 3 & 2 & 1 & 4 & 3 & 3 & 3 & 3 & 4 & 3 & 4 & 3 & 2 & 3 & 3 & 3 & 4 & 61.00 \\
\hline 17 & 3 & 4 & 4 & 3 & 2 & 1 & 4 & 3 & 4 & 3 & 4 & 4 & 3 & 4 & 4 & 4 & 4 & 4 & 4 & 4 & 70.00 \\
\hline 18 & 3 & 3 & 3 & 3 & 3 & 1 & 4 & 3 & 3 & 4 & 3 & 4 & 3 & 4 & 3 & 2 & 3 & 3 & 3 & 3 & 61.00 \\
\hline 19 & 2 & 2 & 3 & 2 & 3 & 1 & 4 & 3 & 3 & 3 & 4 & 4 & 3 & 2 & 3 & 2 & 3 & 3 & 3 & 4 & 57.00 \\
\hline 20 & 4 & 2 & 1 & 3 & 2 & 1 & 4 & 3 & 3 & 3 & 4 & 4 & 2 & 2 & 4 & 2 & 3 & 3 & 3 & 4 & 57.00 \\
\hline 21 & 3 & 2 & 1 & 3 & 3 & 1 & 4 & 3 & 4 & 4 & 4 & 4 & 3 & 3 & 3 & 2 & 3 & 3 & 3 & 4 & 60.00 \\
\hline 22 & 2 & 3 & 1 & 3 & 2 & 1 & 4 & 3 & 3 & 3 & 4 & 4 & 3 & 2 & 3 & 2 & 3 & 3 & 4 & 4 & 57.00 \\
\hline 23 & 2 & 2 & 3 & 3 & 2 & 1 & 4 & 3 & 3 & 3 & 4 & 4 & 3 & 2 & 3 & 2 & 3 & 4 & 3 & 4 & 58.00 \\
\hline 24 & 2 & 2 & 2 & 3 & 2 & 2 & 4 & 3 & 3 & 4 & 4 & 4 & 3 & 2 & 3 & 2 & 3 & 3 & 3 & 4 & 58.00 \\
\hline 25 & 3 & 3 & 3 & 3 & 2 & 2 & 4 & 3 & 3 & 3 & 4 & 4 & 2 & 4 & 3 & 2 & 3 & 3 & 4 & 4 & 62.00 \\
\hline$\ldots$ & 3 & 2 & 2 & 3 & 2 & 1 & 4 & 3 & 3 & 3 & 4 & 4 & 2 & 4 & 3 & 3 & 3 & 3 & 4 & 4 & 60.00 \\
\hline 30 & 3 & 2 & 1 & 3 & 2 & 1 & 4 & 3 & 3 & 4 & 4 & 4 & 3 & 4 & 3 & 3 & 3 & 3 & 4 & 4 & 61.00 \\
\hline 31 & 3 & 2 & 1 & 3 & 2 & 1 & 4 & 3 & 3 & 3 & 4 & 4 & 2 & 2 & 3 & 3 & 3 & 3 & 4 & 4 & 57.00 \\
\hline 32 & 1 & 3 & 3 & 3 & 2 & 1 & 4 & 3 & 3 & 3 & 4 & 4 & 2 & 4 & 3 & 3 & 4 & 3 & 3 & 4 & 60.00 \\
\hline 33 & 3 & 2 & 2 & 3 & 2 & 1 & 4 & 3 & 3 & 4 & 4 & 4 & 2 & 4 & 3 & 3 & 4 & 3 & 4 & 4 & 62.00 \\
\hline$\ldots$ & 3 & 2 & 3 & 3 & 2 & 1 & 4 & 3 & 4 & 3 & 4 & 4 & 3 & 4 & 4 & 3 & 3 & 3 & 3 & 4 & 63.00 \\
\hline 41 & 4 & 3 & 3 & 3 & 2 & 1 & 4 & 3 & 3 & 3 & 4 & 4 & 3 & 4 & 3 & 3 & 3 & 3 & 4 & 4 & 64.00 \\
\hline 42 & 3 & 2 & 2 & 3 & 2 & 1 & 4 & 3 & 3 & 3 & 4 & 4 & 2 & 4 & 4 & 3 & 4 & 3 & 4 & 4 & 62.00 \\
\hline 43 & 3 & 2 & 3 & 3 & 2 & 1 & 4 & 3 & 3 & 3 & 4 & 4 & 3 & 4 & 3 & 3 & 4 & 3 & 3 & 4 & 62.00 \\
\hline 44 & 3 & 2 & 3 & 3 & 2 & 1 & 4 & 3 & 3 & 3 & 4 & 4 & 3 & 4 & 3 & 3 & 4 & 3 & 3 & 4 & 62.00 \\
\hline 45 & 3 & 3 & 3 & 3 & 2 & 1 & 4 & 3 & 3 & 3 & 4 & 4 & 3 & 4 & 4 & 3 & 4 & 3 & 4 & 4 & 65.00 \\
\hline 46 & 3 & 2 & 2 & 3 & 2 & 1 & 4 & 3 & 3 & 3 & 4 & 4 & 2 & 4 & 3 & 3 & 4 & 3 & 3 & 4 & 60.00 \\
\hline 47 & 3 & 2 & 3 & 3 & 2 & 1 & 4 & 3 & 3 & 3 & 4 & 4 & 3 & 4 & 3 & 3 & 3 & 3 & 3 & 4 & 61.00 \\
\hline 48 & 3 & 2 & 3 & 3 & 2 & 1 & 4 & 3 & 3 & 4 & 4 & 4 & 2 & 4 & 3 & 3 & 4 & 3 & 3 & 4 & 62.00 \\
\hline$\ldots$ & 4 & 3 & 2 & 3 & 2 & 1 & 4 & 3 & 3 & 3 & 4 & 4 & 2 & 4 & 3 & 3 & 3 & 3 & 3 & 4 & 61.00 \\
\hline 59 & 3 & 2 & 3 & 3 & 2 & 1 & 4 & 3 & 3 & 3 & 4 & 4 & 3 & 4 & 4 & 3 & 4 & 3 & 3 & 4 & 63.00 \\
\hline 60 & 4 & 3 & 3 & 3 & 2 & 1 & 4 & 3 & 3 & 4 & 4 & 4 & 3 & 4 & 3 & 3 & 4 & 3 & 4 & 4 & 66.00 \\
\hline 61 & 3 & 3 & 3 & 3 & 2 & 1 & 4 & 3 & 3 & 3 & 4 & 4 & 2 & 4 & 3 & 3 & 3 & 3 & 3 & 4 & 61.00 \\
\hline 62 & 4 & 2 & 3 & 3 & 2 & 1 & 4 & 3 & 3 & 3 & 4 & 4 & 2 & 4 & 3 & 3 & 3 & 3 & 3 & 4 & 61.00 \\
\hline 63 & 3 & 3 & 3 & 3 & 2 & 1 & 4 & 3 & 3 & 3 & 4 & 4 & 3 & 4 & 4 & 3 & 3 & 3 & 3 & 4 & 63.00 \\
\hline 64 & 3 & 3 & 3 & 3 & 2 & 1 & 4 & 3 & 3 & 3 & 4 & 4 & 2 & 4 & 3 & 3 & 3 & 3 & 4 & 4 & 62.00 \\
\hline
\end{tabular}




\begin{tabular}{|c|c|c|c|c|c|c|c|c|c|c|c|c|c|c|c|c|c|c|c|c|c|}
\hline 65 & 3 & 3 & 3 & 3 & 2 & 1 & 4 & 3 & 3 & 3 & 4 & 4 & 3 & 4 & 3 & 3 & 3 & 3 & 4 & 4 & 63.00 \\
\hline 66 & 3 & 3 & 3 & 3 & 2 & 1 & 4 & 3 & 3 & 3 & 4 & 4 & 2 & 4 & 3 & 3 & 3 & 3 & 4 & 4 & 62.00 \\
\hline 67 & 3 & 3 & 3 & 3 & 3 & 1 & 4 & 3 & 3 & 3 & 4 & 4 & 2 & 4 & 3 & 3 & 4 & 3 & 3 & 4 & 63.00 \\
\hline 68 & 4 & 3 & 3 & 3 & 2 & 1 & 4 & 3 & 3 & 3 & 4 & 4 & 3 & 4 & 3 & 3 & 4 & 3 & 4 & 4 & 65.00 \\
\hline 69 & 3 & 3 & 3 & 3 & 2 & 1 & 4 & 3 & 3 & 3 & 4 & 4 & 2 & 4 & 3 & 3 & 4 & 3 & 4 & 4 & 63.00 \\
\hline 70 & 3 & 3 & 3 & 3 & 2 & 1 & 4 & 3 & 3 & 3 & 4 & 4 & 3 & 4 & 3 & 3 & 4 & 3 & 3 & 4 & 63.00 \\
\hline 71 & 3 & 3 & 3 & 3 & 2 & 4 & 4 & 3 & 3 & 4 & 4 & 4 & 2 & 4 & 3 & 3 & 4 & 3 & 3 & 4 & 66.00 \\
\hline 72 & 4 & 3 & 3 & 3 & 2 & 1 & 4 & 3 & 3 & 4 & 4 & 4 & 3 & 4 & 3 & 3 & 2 & 3 & 3 & 4 & 63.00 \\
\hline 73 & 3 & 2 & 3 & 3 & 2 & 1 & 4 & 3 & 4 & 3 & 4 & 4 & 3 & 4 & 3 & 3 & 2 & 3 & 3 & 4 & 61.00 \\
\hline 74 & 3 & 3 & 3 & 3 & 2 & 1 & 4 & 3 & 4 & 4 & 4 & 4 & 2 & 4 & 3 & 3 & 4 & 3 & 3 & 4 & 64.00 \\
\hline 75 & 4 & 3 & 3 & 3 & 2 & 1 & 4 & 3 & 3 & 4 & 4 & 4 & 2 & 4 & 3 & 3 & 3 & 3 & 3 & 4 & 63.00 \\
\hline 76 & 4 & 3 & 3 & 3 & 2 & 1 & 4 & 3 & 3 & 4 & 4 & 4 & 3 & 4 & 4 & 3 & 3 & 3 & 3 & 4 & 65.00 \\
\hline 77 & 3 & 2 & 3 & 3 & 2 & 2 & 4 & 3 & 3 & 4 & 4 & 4 & 3 & 4 & 4 & 3 & 3 & 3 & 3 & 4 & 64.00 \\
\hline 78 & 3 & 2 & 2 & 3 & 2 & 2 & 4 & 3 & 4 & 4 & 4 & 4 & 2 & 4 & 3 & 3 & 3 & 3 & 3 & 4 & 62.00 \\
\hline 79 & 3 & 2 & 3 & 3 & 2 & 2 & 4 & 3 & 3 & 3 & 4 & 4 & 3 & 4 & 3 & 3 & 3 & 3 & 3 & 4 & 62.00 \\
\hline 80 & 3 & 2 & 3 & 3 & 2 & 1 & 4 & 3 & 3 & 4 & 4 & 4 & 3 & 4 & 3 & 3 & 3 & 3 & 3 & 4 & 62.00 \\
\hline 81 & 3 & 3 & 3 & 3 & 2 & 2 & 4 & 3 & 3 & 4 & 4 & 4 & 2 & 4 & 3 & 3 & 3 & 3 & 3 & 4 & 63.00 \\
\hline 82 & 3 & 3 & 3 & 3 & 2 & 1 & 4 & 3 & 3 & 4 & 4 & 4 & 3 & 3 & 3 & 3 & 3 & 3 & 3 & 4 & 62.00 \\
\hline 83 & 3 & 2 & 3 & 3 & 2 & 1 & 4 & 3 & 3 & 4 & 4 & 4 & 2 & 3 & 4 & 3 & 3 & 3 & 3 & 4 & 61.00 \\
\hline 84 & 3 & 3 & 3 & 3 & 2 & 1 & 4 & 3 & 4 & 3 & 4 & 4 & 3 & 4 & 3 & 3 & 4 & 3 & 4 & 4 & 65.00 \\
\hline 85 & 3 & 2 & 2 & 3 & 2 & 1 & 4 & 3 & 4 & 4 & 4 & 4 & 3 & 4 & 3 & 3 & 3 & 3 & 4 & 4 & 63.00 \\
\hline 86 & 4 & 3 & 3 & 3 & 2 & 2 & 4 & 3 & 3 & 4 & 4 & 4 & 3 & 4 & 3 & 3 & 3 & 3 & 4 & 4 & 66.00 \\
\hline 87 & 3 & 2 & 3 & 3 & 2 & 1 & 4 & 3 & 3 & 3 & 4 & 4 & 3 & 4 & 3 & 3 & 3 & 3 & 4 & 4 & 62.00 \\
\hline 88 & 3 & 3 & 3 & 3 & 2 & 2 & 4 & 3 & 3 & 4 & 4 & 4 & 3 & 4 & 3 & 3 & 3 & 3 & 3 & 4 & 64.00 \\
\hline 89 & 4 & 2 & 3 & 3 & 2 & 2 & 4 & 3 & 3 & 3 & 4 & 4 & 3 & 4 & 3 & 3 & 3 & 3 & 4 & 4 & 64.00 \\
\hline 90 & 3 & 2 & 3 & 3 & 2 & 1 & 4 & 3 & 3 & 3 & 4 & 4 & 2 & 4 & 3 & 3 & 3 & 3 & 4 & 4 & 61.00 \\
\hline 91 & 3 & 2 & 3 & 3 & 2 & 2 & 4 & 3 & 3 & 3 & 4 & 4 & 2 & 4 & 3 & 3 & 3 & 3 & 3 & 4 & 61.00 \\
\hline 92 & 4 & 3 & 3 & 3 & 2 & 2 & 4 & 3 & 4 & 3 & 4 & 4 & 2 & 4 & 3 & 3 & 2 & 3 & 3 & 4 & 63.00 \\
\hline 93 & 3 & 3 & 3 & 3 & 2 & 2 & 4 & 3 & 3 & 3 & 4 & 4 & 2 & 4 & 3 & 3 & 3 & 3 & 3 & 4 & 62.00 \\
\hline 94 & 3 & 2 & 3 & 3 & 2 & 2 & 4 & 3 & 3 & 4 & 4 & 4 & 2 & 4 & 3 & 3 & 3 & 3 & 3 & 4 & 62.00 \\
\hline$\ldots$ & 3 & 3 & 3 & 3 & 2 & 1 & 3 & 3 & 3 & 3 & 3 & 4 & 2 & 4 & 3 & 3 & 3 & 3 & 4 & 4 & 60.00 \\
\hline 106 & 3 & 2 & 3 & 3 & 2 & 2 & 3 & 3 & 3 & 3 & 4 & 4 & 2 & 4 & 4 & 3 & 3 & 3 & 3 & 4 & 61.00 \\
\hline 107 & 3 & 3 & 3 & 3 & 2 & 1 & 3 & 3 & 3 & 3 & 4 & 4 & 2 & 4 & 3 & 3 & 3 & 3 & 3 & 4 & 60.00 \\
\hline 108 & 3 & 3 & 3 & 3 & 2 & 1 & 3 & 3 & 3 & 3 & 4 & 4 & 2 & 1 & 3 & 1 & 3 & 3 & 3 & 4 & 55.00 \\
\hline 109 & 4 & 3 & 3 & 3 & 2 & 1 & 3 & 3 & 4 & 3 & 4 & 4 & 2 & 4 & 3 & 3 & 3 & 3 & 3 & 4 & 62.00 \\
\hline 110 & 3 & 3 & 3 & 3 & 2 & 1 & 3 & 3 & 3 & 3 & 4 & 4 & 3 & 4 & 3 & 3 & 3 & 3 & 3 & 4 & 61.00 \\
\hline 111 & 3 & 2 & 3 & 3 & 2 & 1 & 3 & 3 & 4 & 3 & 3 & 4 & 2 & 2 & 3 & 3 & 3 & 3 & 3 & 4 & 57.00 \\
\hline 112 & 3 & 2 & 3 & 3 & 3 & 1 & 3 & 1 & 3 & 3 & 3 & 4 & 1 & 1 & 3 & 3 & 2 & 3 & 3 & 4 & 52.00 \\
\hline 113 & 2 & 4 & 3 & 1 & 2 & 1 & 3 & 3 & 3 & 3 & 3 & 4 & 2 & 1 & 3 & 3 & 3 & 3 & 3 & 4 & 54.00 \\
\hline 114 & 3 & 2 & 2 & 3 & 2 & 1 & 3 & 3 & 3 & 3 & 4 & 4 & 2 & 1 & 3 & 3 & 3 & 3 & 3 & 4 & 55.00 \\
\hline 115 & 3 & 3 & 3 & 3 & 2 & 1 & 3 & 3 & 3 & 3 & 3 & 4 & 2 & 1 & 3 & 3 & 3 & 3 & 3 & 4 & 56.00 \\
\hline 116 & 3 & 3 & 2 & 3 & 4 & 2 & 3 & 3 & 3 & 2 & 3 & 4 & 2 & 1 & 2 & 3 & 1 & 3 & 3 & 2 & 52.00 \\
\hline 117 & 3 & 3 & 4 & 3 & 3 & 1 & 1 & 3 & 3 & 3 & 3 & 4 & 2 & 2 & 3 & 3 & 3 & 3 & 3 & 4 & 57.00 \\
\hline 118 & 3 & 3 & 3 & 3 & 1 & 1 & 3 & 3 & 3 & 3 & 3 & 1 & 4 & 1 & 1 & 3 & 3 & 3 & 3 & 2 & 50.00 \\
\hline 119 & 1 & 3 & 1 & 3 & 3 & 1 & 4 & 2 & 1 & 1 & 3 & 4 & 3 & 1 & 3 & 3 & 3 & 3 & 3 & 4 & 50.00 \\
\hline 120 & 3 & 1 & 2 & 4 & 2 & 1 & 2 & 3 & 2 & 3 & 4 & 2 & 2 & 1 & 3 & 3 & 3 & 3 & 3 & 4 & 51.00 \\
\hline Mode & 3.0 & 2.0 & 3.0 & 3.0 & 2.0 & 1.0 & 4.0 & 3.0 & 3.0 & 3.0 & 4.0 & 4.0 & 2.0 & 4.0 & 3.0 & 3.0 & 3.0 & 3.0 & 3.0 & 4.0 & \\
\hline Median & 3.0 & 2.0 & 3.0 & 3.0 & 2.0 & 1.0 & 4.0 & 3.0 & 3.0 & 3.0 & 4.0 & 4.0 & 2.0 & 4.0 & 3.0 & 3.0 & 3.0 & 3.0 & 3.0 & 4.0 & \\
\hline $\begin{array}{c}\text { 1st } \\
\text { quartile }\end{array}$ & 1.00 & & & & & & & & & & & & & & & & & & & & \\
\hline $\begin{array}{c}\text { 3rd } \\
\text { quartile }\end{array}$ & 4.00 & & & & & & & & & & & & & & & & & & & & \\
\hline & & & & & & & & & & & & & & & & & & & & & \\
\hline
\end{tabular}

Source: Prepared by the author. 
was comprised of students and professors of the School of Economic Sciences of UNMSM, considering a total of 60 professors and 1800 students. The prototype used is equivalent to $17.61 \%$ of the population, with a margin of error of $5 \%$ and a confidence level of $95 \%$; therefore, a sample of 120 respondents, both professors and students, was considered for this survey.

\section{Hypothesis formulation}

\section{General hypothesis}

The application of appropriate ICTs influences the quality of university learning.

\section{Specific hypotheses}

- Appropriate ICTs improve the quality of university learning.

- The level of learning quality influences the learning process of university students.

\section{RESULTS}

Table 1, contained in the Annex, shows the results obtained from the questionnaire addressed to 120 respondents.

\section{Reliability Analysis}

Cronbach's alpha is used in this study, as according to Ledesma (2004) it makes it possible to estimate the consistency and reliability of a questionnaire. Consequently, a value of 0.744 was found, which is within the 0.7-0.8 range, which defines the questionnaire used as a reliable instrument. The result was obtained using IBM SPSS Statistics 24.0 software. The results of the reliability statistics obtained from the Cronbach's analysis are shown in Table 2.

Table 2. Cronbach's analysis

\begin{tabular}{|l|c|}
\hline \multicolumn{2}{|c|}{ Reliability statistics } \\
\hline Cronbach's alpha & No. of items \\
\hline 0.744 & 20 \\
\hline
\end{tabular}

Source: Prepared by the author.

\section{Exploratory Factor Analysis}

An exploratory factor analysis of principal components with varimax rotation was performed. Additionally, Barlett's test for sphericity helped determine whether the matrix was factorable or not. Results are shown in Table 3.

For the sample of this study, the Kaiser-Meyer-Olkin (KMO) index yielded a value of 0.679 . Since the result is greater than 0.500 , factor analysis was recommended. Bartlett's test for sphericity is highly sig- nificant $(p=0.000)$, indicating that there are relevant statistical relationships among certain variables.

Table 3. Kaiser-Meyer-Olkin and Barlett tests.

\begin{tabular}{|l|l|c|}
\hline \multicolumn{2}{|l|}{$\begin{array}{l}\text { Kaiser-Meyer-Olkin Measure of Sampling } \\
\text { Adequacy }\end{array}$} & 0.679 \\
\hline \multirow{2}{*}{$\begin{array}{l}\text { Bartlett's test for } \\
\text { sphericity }\end{array}$} & Approximate Chi-Square & 616.242 \\
\cline { 2 - 3 } & Degrees of freedom & 190 \\
\cline { 2 - 3 } & Significance & 0.000 \\
\hline
\end{tabular}

Source: SPSS.

\section{Hypothesis Analysis}

Following Cánovas, Pérez and Tuya (2009), the Excel system was used to calculate the correlation coefficient for the general hypothesis, obtaining a value of 0.543 , which indicates the hypothesis is valid. Hence:

- The calculation of the correlation coefficient for the general hypothesis obtained $D=0,543$.

- The calculation of the correlation coefficient for the specific hypothesis (related to ICTs) obtained $D=0,702$, indicating the hypothesis is valid.

- The calculation of the correlation coefficient for the specific hypothesis (level of learning) obtained $D=0,612$, indicating that the specific hypothesis is valid.

- A result $>0.5$ was obtained in all cases. This indicates that there is correlation between the variables, thus confirming the posed hypotheses.

The process stages were as follows: sample selection, drafting of the instrument, questionnaire generation for professors and students, printing and revision of the instrument, procurement of the respective permits from the Dean and head of the School of Economic Sciences of UNMSM, administration of the survey with the collaboration of students of Universidad César Vallejo (UCV), data entry to the Excel system, statistical descriptive analysis using Likert scale, processing of results and report preparation.

\section{CONCLUSIONS}

- The posed hypotheses were contrasted with the results obtained from the questionnaire addressed to professors and students of the School of Economic Sciences of UNMSM, where consistency statistics (Cronbach's alpha) and Spearman' rank order correlation coefficient were used for each class interval. 
Similarly, descriptive statistics by means of mode, median and quartiles were used.

- As shown in Table 1 (see Annex), the variables analyzed were ICT and quality of learning. On this basis, it is concluded that the use of e-mail has become a necessity for professors and pupils. In addition, classroom-based teaching continues to be more used and offers better results. Conversely, projectors are essential in didactic and educational activities.

\section{Recommendations}

- Request that professors keep up to date regarding the use of educational software.

- Increase the use of videoconferences.

- Use online examinations more frequently.

- Enhance the filtering of information provided to students.

- Increase the dissemination of new technologies among students.

- Emphasize the importance of education in the Peruvian State budget.

- Emphasize the importance of funds earmarked for education in the Peruvian State budget.

\section{REFERENCES}

[1] Barriga, C. (2009). Investigación Educacional A. Texto autoinstructivo. Lima, Peru: Universidad Nacional Mayor de San Marcos.

[2] Bustos, A. \& Román M. (2011). La importancia de evaluar la incorporación y el uso de las TIC en educación. Revista Iberoamericana de Evaluación Educativa, 4(2), 3-7. Retrieved from https://revistas.uam.es/index.php/riee/ article/view/4452/4879

[3] Cabero, J. (2014). Formación del profesorado universitario en TIC. Aplicación del método Delphi para la selección de los contenidos formativos. Educación XX1, 17(1), 111-132. Retrieved from http://revistas.uned.es/index. php/educacionXX1/article/view/10707/11301

[4] Cánovas, A., Martínez, R., Pérez, A. \& Tuya, L. (2009). El coeficiente de correlación de los rangos de Spearman. Caracterización. Revista Habanera de Ciencias Médicas, 8(2). Retrieved from http://scielo.sld.cu/scielo.php?script=sci_ arttext\&pid=S1729-519X2009000200017

[5] Cuenca, R. (ed.) (2015). La educación universitaria en el Perú. Democracia, expansión y desigualdades. Lima, Peru: Instituto de Estudios Peruanos.

[6] Fuente, J. de la \& Justicia, F. (2003). Escala de estrategias de aprendizaje ACRA-Abreviada para alumnos universitarios. Revista Electrónica de Investigación Psicoeducativa y Psicopedagógica, 1(2), 139-158. Retrieved from http://www.investigacion-psicopedagogica.org/ revista/articulos/2/espannol/Art_2_16.pdf

[7] Álvarez, P. (2016, December 6). Informe PISA. La educación española se estanca en ciencias y matemáticas y mejora levemente en lectura. El País. Retrieved from https://elpais.com/politica/2016/12/05/ actualidad/1480950645_168779.html.

[8] Ledesma, R. (2004). AlphaCl: un programa de cálculo de intervalos de confianza para el coeficiente alfa de Cronbach. Psico-USF, 9(1), 31-37. Retrieved from http://pepsic.bvsalud. org/pdf/psicousf/v9n1/v9n1a05.pdf

[9] Martí, J. (2014, June 6). Las TIC en Finlandia. Xarxatix. Retrieved from http://www.xarxatic. com/las-tic-en-finlandia/

[10] Morales, M., Trujillo, J. \& Raso, F. (2015). Percepciones acerca de la integración de las TIC en el proceso de enseñanza-aprendizaje de la universidad. Pixel-Bit. Revista de Medios y Educación, (46), 103-117. Retrieved from https://idus.us.es/xmlui/bitstream/ handle/11441/45403/PERCEPCIONES\%20 A C E R C A \% 20 D E \% 20 L A \% 20 INTEGRACI\%C3\%93N\%20DE\%20LAS\%20 TIC\%20EN.pdf?sequence=1\&isAllowed=y

[11] Nakano T., Garret, P., Vásquez, A. \& Mija, Á. (2014). La integración de las TIC en la educación superior: reflexiones y aprendizajes a partir de la experiencia PUCP. Blanco \& Negro. Revista sobre Docencia Universitaria, 4(2), 65-76. Retrieved from http://revistas.pucp. edu.pe/index.php/enblancoynegro/article/ view/8936/9344

[12] Rodrigues, L. M. A. R. (2015). Os jogos como método de aprendizagem de vocabulário nas aulas de espanhol como língua estrangeira (ELE). (Doctoral Thesis). Retrieved from https:// repositorio.ucp.pt/handle/10400.14/18809.

[13] Radio Programas del Perú (2012, August 30). Brasil quiere convertirse en el tercer mercado mundial de las TIC. Retrieved from https://rpp. pe/lima/actualidad/brasil-quiere-convertirseen-el-tercer-mercado-mundial-de-las-ticnoticia-517195. 